\title{
Feeding practices in early life and later intake of fruit and vegetables among Japanese toddlers: the Osaka Maternal and Child Health Study
}

\author{
Hitomi Okubo ${ }^{1, *}$, Yoshihiro Miyake ${ }^{2}$, Satoshi Sasaki ${ }^{3}$, Keiko Tanaka $^{2}$ and Yoshio Hirota ${ }^{4}$ \\ 'Department of Health Promotion, National Institute of Public Health, 2-3-6 Minami, Wako-shi, Saitama 351-0197, \\ Japan: ${ }^{2}$ Department of Epidemiology and Preventive Medicine, Ehime University Graduate School of Medicine, \\ Ehime, Japan: ${ }^{3}$ Department of Social and Preventive Epidemiology, School of Public Health, University of Tokyo, \\ Tokyo, Japan: ${ }^{4}$ Clinical Epidemiology Research Center, Medical Co., LTA, Fukuoka, Japan
}

Submitted 26 January 2015: Final revision received 27 March 2015: Accepted 22 April 2015: First published online 3 June 2015

\begin{abstract}
Objective: A growing body of evidence from Western countries shows that infant feeding practices are associated with later childhood dietary habits, but little is known about these relationships in non-Western countries with different food cultures. We examined the association of breast-feeding duration and age at introduction of solid foods with later intake of fruit and vegetables among Japanese toddlers.

Design: Information on breast-feeding duration, age at introduction of solid foods and child's intake frequency of fruit and vegetables were collected with a selfadministered questionnaire at 16-24 months postpartum. Logistic regression analysis was used to calculate odds ratios of low intake $(<1$ time/d) of fruit or vegetables for each infant feeding practice.

Setting: Japan.

Subjects: Japanese mother-child pairs ( $n$ 763) from a prospective birth cohort study.

Results: Neither breast-feeding duration nor age at introduction of solid foods was associated with fruit intake at 16-24 months of age. Breast-feeding duration, but not age at introduction of solid foods, was associated with later intake of vegetables. When breast-feeding duration was categorized into two groups with the cut-off at 6 months, children who were breast-fed for $\geq 6$ months had a significantly decreased risk of low intake of vegetables (OR $=0.53 ; 95 \%$ CI 0.34 , $0 \cdot 84$ ) than those breast-fed for $<6$ months. This association was independent of potential confounders including maternal education and maternal vegetable intake (OR=0.59; $95 \%$ CI $0 \cdot 36,0 \cdot 97$ ).

Conclusions: This finding suggests that $\geq 6$ months of breast-feeding may prevent low intake of vegetables in early childhood among Japanese toddlers.
\end{abstract}

\author{
Keywords \\ Breast-feeding \\ Infant nutrition \\ Fruit and vegetable intake
}

Infancy is a critical period of dramatic dietary transition, from an entirely milk-based diet to a diet based on solid foods with a variety of family foods from all food groups. Adequate nutrition in infancy not only affects short- and long-term health status ${ }^{(1,2)}$, but is also known to influence the formation of the child's dietary habits and food preferences $^{(3,4)}$. It is evident that a diet high in fruit and vegetables in early childhood may lead to lifelong high intake of fruit and vegetables, which has many beneficial effects on weight control and other health outcomes in later life ${ }^{(5-8)}$. However, the proportion of young children who consume less than the recommended amounts of fruit and vegetables is increasing and is becoming a worldwide public health issue ${ }^{(9-11)}$. In Japan, $62.7 \%$ and $35.0 \%$ of children aged 1-4 years consumed fruit and vegetables less than once daily, respectively ${ }^{(12)}$. Understanding the modifiable factors involved in determining whether a child's early-life diet is high in fruit and vegetables and identifying children with unhealthy dietary habits are therefore particularly important steps in preventing the onset of diet-related disease in later life.

The influence of variation in infant nutrition on later dietary habits and food preferences is beginning to receive considerable attention. In particular, breast-feeding is one of a child's initial opportunities for early exposure to different flavours. Some observational studies have found associations 
between longer duration of breast-feeding and higher intake levels of fruit and/or vegetables in later childhood ${ }^{(13-17)}$. In addition, some researchers have suggested that there is a 'sensitive' period for the introduction of tastes between 4 and 6 months of age in which infants more readily accept a wide range of tastes ${ }^{(18)}$. This implies that the age at which new tastes are introduced could be important in determining the child's subsequent acceptance of new foods and in shaping dietary habits. There is some evidence showing that an early introduction to fruit and vegetables is associated with an increased intake of fruit and vegetables in later childhood ${ }^{(16)}$, but the significance of this relationship is inconsistent across studies ${ }^{(14,15)}$. It should be noted that the previous studies on this topic have been conducted predominantly in Western countries; in fact, we are unaware of any comparable research reported in Asian countries including Japan, although the food cultures of Asian and Western peoples are quite different. It will be necessary to accumulate studies of this kind from various regions with different social and cultural backgrounds in order to obtain reliable scientific evidence on the relationship between infant nutrition and children's later diets.

In the present study we examine whether infant nutrition (i.e. duration of breast-feeding and age at introduction of solid foods) influences later intake of fruit and vegetables in a group of Japanese toddlers aged 16-24 months. We considered the effects of several potential factors that have been suggested to influence children's diets, including maternal socio-economic status and maternal fruit and vegetable intake ${ }^{(13-15)}$.

\section{Materials and methods}

\section{Study population and procedure}

The Osaka Maternal and Child Health Study (OMCHS) is a prospective cohort study that investigates preventive and risk factors for maternal and child health problems. The principal objective of the OMCHS is to clarify risk factors for childhood allergic disorders. A detailed description of the rationale, study design and protocol has been published elsewhere ${ }^{(19,20)}$. Briefly, between November 2001 and March 2003, all pregnant women in Neyagawa City, Osaka Prefecture, Japan were asked to participate. Of 3639 pregnant women, 627 (17.2\%) took part in the study. An additional 375 pregnant women living in other municipalities were enrolled between December 2001 and November 2003. Baseline assessment of the OMCHS was primarily conducted using a set of two self-administered questionnaires on dietary habits and a wide range of lifestyle behaviours. A self-administered questionnaire was also used in each of the second and third surveys, conducted after the birth of the children. The participants returned their completed questionnaires for each survey to the data management centre. Research technicians confirmed missing or illogical data by telephone interview. A total of 1002 pregnant women at 5-39 weeks of gestation completed the baseline survey. Of these 1002 women, 867 and 763 mother-child pairs participated in the second (at 29 months postpartum) and third (at 16-24 months postpartum) surveys, respectively. In the case of multiple births ( $n 7$ ), one child per mother was selected at random to be included in the analyses. Data on 763 mother-child pairs were used for the present analysis.

The OMCHS was conducted according to the guidelines of the Declaration of Helsinki and approved by the ethics committee of the Osaka City University School of Medicine. Written informed consent was obtained from all participating women.

\section{Maternal data}

At the baseline survey during pregnancy, we collected information on maternal age, gestational age at enrolment (weeks), education ( $<13$ years (high school or less), 13-14 years (technical or professional school) or $\geq 15$ years (university or more)) and annual household income (<4000 000, $4000000-5999999$ or $\geq 6000000$ Japanese yen/year). Diet during the preceding month was assessed using a validated, self-administered dietary history questionnaire (DHQ) ${ }^{(21-27)}$. Estimates of daily intake for 150 food and beverage items as well as for energy, nutrients and alcohol were calculated using an ad hoc computer algorithm for the DHQ, which was based on the Standard Tables of Food Composition in Japan ${ }^{(28)}$. Validity of the DHQ with respect to commonly studied nutritional factors has been investigated in several previous studies, using a dietary record $^{(21,24,25)}, 24 \mathrm{~h}$ urine excretion ${ }^{(22)}$ and serum biomarkers $^{(23,26,27)}$. In a previous study of ninety-two Japanese women aged 31-69 years, the Pearson correlation coefficients between the DHQ and $16 \mathrm{~d}$ semi-weighed dietary record were 0.27 to 0.87 for energy-providing nutrients, 0.39 to 0.71 for other nutrients ${ }^{(25)}, 0.40$ for fruit and 0.56 for total vegetables ${ }^{(24)}$. In other studies of Japanese pregnant women, Spearman correlation coefficients between the DHQ-assessed intakes and serum concentrations were 0.29 for folate, 0.22 for vitamin $\mathrm{B}_{12}{ }^{(26)}, 0.25$ for $\beta$-carotene and 0.32 for vitamin $\mathrm{C}^{(27)}$. These data suggest the satisfactory validity of the DHQ in terms of these dietary variables. However, its validity with regard to other dietary variables has not been investigated among Japanese pregnant women. Self-reported body height (in centimetres) and weight (in kilograms) were also obtained from the DHQ, from which BMI was calculated as body weight divided by the square of body height $\left(\mathrm{kg} / \mathrm{m}^{2}\right)$. In the second survey, completed after delivery, information on parity and smoking status in pregnancy (never smoked, stopped smoking in pregnancy or smoked throughout pregnancy) was obtained.

\section{Children's data}

Information on baby's sex, birth weight (in grams) and gestational age at birth was obtained by the self-administered questionnaire composing the second survey ${ }^{(20)}$. In the third 
survey, conducted at 16-24 months postpartum, we obtained details of feeding practices in early life (i.e. breast-feeding duration and the age in months at which solid foods were introduced). The duration of breast-feeding was defined as the period during which children had received breast milk, irrespective of exclusiveness, and categorized into four groups: $<6,6-11,12-17$ or $\geq 18$ months. These categories were chosen based on the 25th, 50th and 75th percentiles of breast-feeding duration in the study sample because no clear recommendation on breast-feeding duration exists in Japan. The guideline of weaning for infants in Japan recommends gradual introduction of solid foods from the age of 5 to 6 months ${ }^{(29)}$. Based on this guideline, the ages of the children when solid foods were introduced were categorized into three groups: $<5,5-5.9$ or $\geq 6$ months. Information on the child's dietary habits during the preceding month was obtained at the third survey from the mother in terms of the consumption frequency of twenty-one selected food and beverage items without specification of portion size $^{(30)}$. Eight predefined frequency categories ranging from 'less than once per month' to 'two or more times per day' were used. For predefined frequency categories, the midpoint of each category was assumed to be the most likely consumption (e.g. reported consumption of '4-6 times/ week' was calculated as '5 times/week'). All reported frequency categories for each item were then converted to a daily consumption value. Of the foods listed on the questionnaire, three items were specifically related to fruit and vegetable intake: fruit, vegetables and vegetables from commercial baby foods. Daily intake of vegetables, as the total consumption frequency (in times per day), was calculated as the sum of vegetables and vegetables from commercial baby foods.

\section{Statistical analysis}

Descriptive data are presented as mean values and standard deviations for continuous variables and as percentages of the participants for categorical variables. Differences in group characteristics according to categories of feeding practices in early life were examined with the $\chi^{2}$ test for categorical data or ANOVA for continuous data. Univariate and multiple logistic regression analyses were used to examine the relationships between feeding practices in early life and later intake of fruit and vegetables. In the present study, we calculated odds ratios and $95 \%$ confidence intervals of low intake frequency of fruit and vegetables (less than once daily) for each category of infant feeding practices to clarify how these practices affect the risk of forming unhealthy dietary habits in early childhood. Because no dietary recommendation on fruit and vegetable intake for young children exists in Japan, the cut-off point was chosen based on a literature review of the earlier studies ${ }^{(14,16,17)}$. The lowest category of each variable of infant feeding practices served as the reference group.
For the logistic regression analysis, we controlled for the effects of the following potential confounding factors: maternal factors were age at enrolment, BMI, education, annual household income, smoking status during pregnancy, and fruit and vegetable intake during pregnancy; child factors were sex, birth weight and age at the third survey. Because the two early-life feeding practices studied here (breast-feeding duration and the age of introduction of solid foods) are strongly correlated, they were entered simultaneously in the regression models. Tests for trend associations were based on continuously distributed variables and adjusted for potential confounders. All statistical analyses were performed using the SAS statistical software package version 9.4. All reported $P$ values are two-tailed and $P<0.05$ was considered to be statistically significant.

\section{Results}

\section{Characteristics of the study population}

Characteristics of the 763 mother-child pairs are shown in Table 1. Compared with other mothers in the OMCHS cohort ( $n$ 239), those included in the analyses were slightly older at enrolment $(P<0.001)$; tended to have a younger gestational age $(P=0 \cdot 02)$, a higher level of education $(P<0 \cdot 001)$ and a higher annual household income $(P<0.001)$; and were more likely to be primiparous $(P<0 \cdot 001)$ and less likely to smoke at baseline $(P<0 \cdot 001)$. There were no differences in maternal height, BMI, energy intake, or fruit and vegetable intake during pregnancy between the mothers studied and the remaining mothers. Slightly fewer than $25 \%$ of children were breast-fed for $<6$ months, while another $24 \%$ were breast-fed for $\geq 18$ months. The introduction of solid foods usually occurred at 5 months of age $(43.5 \%)$ in the present sample. At $18-24$ months of age, $52.0 \%$ and $12.3 \%$ of the children in the present study were at risk of consuming less than one serving daily of fruit and vegetables, respectively.

\section{Associations between feeding practices in early life and participant characteristics}

Characteristics of the mother-child pairs according to four categories of breast-feeding duration and three categories of age at introduction of solid foods are shown in the online supplementary material, Supplemental Tables 1 and 2, respectively. Compared with mothers who breast-fed for $<6$ months, those who breast-fed for $\geq 18$ months were older, tended to have a higher level of education and a higher annual household income, were less likely to smoke during pregnancy and introduced solid foods later (Supplemental Table 1 , all $P<0 \cdot 05$ ). A significant difference was observed between breast-feeding duration and child's vegetable intake at 18 months of age: children who were breast-fed for 6-11 months consumed more vegetables than 
Table 1 Characteristics of the mother-child pairs in OMCHS $(n 763)^{*}$

\begin{tabular}{|c|c|c|}
\hline & \multicolumn{2}{|c|}{ Value } \\
\hline & Mean & SD \\
\hline \multicolumn{3}{|l|}{ Mother } \\
\hline Age at enrolment (years) & $30 \cdot 0$ & 4.0 \\
\hline Gestational age at enrolment (weeks) & $17 \cdot 7$ & 6.7 \\
\hline Height $(\mathrm{cm})$ & $158 \cdot 3$ & 4.9 \\
\hline BMI at enrolment $\left(\mathrm{kg} / \mathrm{m}^{2}\right)$ & 21.4 & $2 \cdot 8$ \\
\hline Primiparous (\%) & \multicolumn{2}{|c|}{$50 \cdot 1$} \\
\hline \multicolumn{3}{|l|}{ Education (years, \%) } \\
\hline$<13$ & \multicolumn{2}{|c|}{$27 \cdot 8$} \\
\hline $13-14$ & \multicolumn{2}{|c|}{$43 \cdot 1$} \\
\hline$\geq 15$ & \multicolumn{2}{|c|}{$29 \cdot 1$} \\
\hline \multicolumn{3}{|l|}{ Family income (Japanese yen/year, \%) } \\
\hline$<4000000$ & \multicolumn{2}{|c|}{$27 \cdot 7$} \\
\hline $4000000-5999999$ & \multicolumn{2}{|c|}{$41 \cdot 0$} \\
\hline$\geq 6000000$ & \multicolumn{2}{|c|}{$31 \cdot 3$} \\
\hline \multicolumn{3}{|l|}{ Smoking status in pregnancy (\%) } \\
\hline Never smoking & \multicolumn{2}{|c|}{$87 \cdot 3$} \\
\hline Stopped smoking & \multirow{2}{*}{\multicolumn{2}{|c|}{$\begin{array}{l}6.4 \\
6.3\end{array}$}} \\
\hline Continued smoking & & \\
\hline \multicolumn{3}{|l|}{ Dietary intake during pregnancy } \\
\hline Energy intake (kJ/d) & 7629 & 1924 \\
\hline Fruit $(\mathrm{g} / \mathrm{d})$ & 110 & 96 \\
\hline Total vegetables $(\mathrm{g} / \mathrm{d})$ & 161 & 86 \\
\hline \multicolumn{3}{|l|}{ Child } \\
\hline Sex (boy, \%) & \multicolumn{2}{|c|}{$52 \cdot 8$} \\
\hline Gestational age (weeks) & $39 \cdot 1$ & 1.6 \\
\hline Birth weight $(g)$ & 3071 & 415 \\
\hline \multicolumn{3}{|l|}{ Breast-feeding duration (months, \%) } \\
\hline$<6$ & \multicolumn{2}{|c|}{$24 \cdot 8$} \\
\hline $6-11$ & \multicolumn{2}{|c|}{$23 \cdot 3$} \\
\hline $12-17$ & \multicolumn{2}{|c|}{$27 \cdot 7$} \\
\hline$\geq 18$ & \multicolumn{2}{|c|}{$24 \cdot 3$} \\
\hline \multicolumn{3}{|c|}{ Age at introduction to solid food (months, \%) } \\
\hline$<5$ & \multirow{3}{*}{\multicolumn{2}{|c|}{$\begin{array}{l}14 \cdot 7 \\
43 \cdot 5 \\
41 \cdot 8\end{array}$}} \\
\hline $5-5.9$ & & \\
\hline$\geq 6$ & & \\
\hline Age at dietary assessment (months) & $19 \cdot 7$ & $1 \cdot 1$ \\
\hline Body weight at 18 months $(\mathrm{kg})$ & $10 \cdot 7$ & $1 \cdot 1$ \\
\hline Height at 18 months $(\mathrm{cm})$ & 81.6 & 3.0 \\
\hline Fruit consumption (times/d) & 0.9 & 0.7 \\
\hline Vegetable consumption (times/d) & 1.9 & 0.8 \\
\hline
\end{tabular}

OMCHS, Osaka Maternal and Child Health Study.

*Values are means and standard deviations or percentages.

did those who were breast-fed for $<6$ months $(P<0 \cdot 05)$. The mothers who introduced solid foods to their children after 6 months of age were older and breast-fed for longer than those who introduced solid foods before 5 months of age (all $P<0.05$ in Supplemental Table 2). The children who began eating solid foods at 5 months of age consumed significantly more vegetables at 16-24 months of age than did those who began eating solid foods before 5 months of age $(P<0 \cdot 05)$.

\section{Associations of feeding practices in early life with later intake of fruit and vegetables}

Table 2 shows the association of breast-feeding duration, the timing of introduction of solid foods and potential confounders with low intake (less than once daily) of fruit and vegetables at 16-24 months of age. Neither breastfeeding duration nor age at introduction of solid foods was associated with the risk of low intake frequency of fruit at 16-24 months of age. In contrast, children who were breast-fed for $\geq 6$ months had significantly lower odds of consuming less than one serving daily of vegetables than were those who were breast-fed for $<6$ months, but a linear trend was not observed ( $P$ for trend $=0 \cdot 13$ ). The lower risks among children breast-fed for $12-17$ or $\geq 18$ months were no longer observed after adjustment for potential maternal and child confounders. The decreased risk of low vegetable intake remained only among children who were breast-fed for 6-11 months. When we categorized breast-feeding duration into two groups using 6 months as the cut-off, breast-feeding for $\geq 6$ months was significantly associated with a decreased risk of low intake of vegetables at 16-24 months of age (crude OR $=0.53$; $95 \%$ CI $0 \cdot 34,0 \cdot 84$ ). Adjustment for potential confounders did not modify the results (adjusted OR $=0.59 ; 95 \% \mathrm{CI}$ $0 \cdot 36,0 \cdot 97)$. No association was observed between age at introduction of solid foods and later intake of vegetables.

\section{Associations of other potential confounders with later intake of fruit and vegetables}

Among the potential confounders examined, maternal education and maternal fruit and vegetable intake during pregnancy were common and strong risk factors related to children's fruit and vegetable intake at 18-24 months of age. Children who were older at dietary assessment and whose mothers had stopped smoking during pregnancy had an increased risk of low intake of fruit, whereas children whose mothers had a higher level of education, higher annual family income and higher fruit intake during pregnancy had a reduced risk of low intake of fruit. On the other hand, higher level of education and vegetable intake during pregnancy were directly associated with a reduced risk of low vegetable intake by children at 16-24 months of age.

\section{Discussion}

The main finding of the current study was that breastfeeding duration may influence later vegetable intake among Japanese toddlers, as observed in the previous Western studies, whereas age at introduction of solid foods did not have an obvious effect on toddlers' fruit or vegetable intake. We found that children who were breastfed for $\geq 6$ months had a significantly reduced risk of low intake frequency of vegetables than those who were breast-fed for $<6$ months, suggesting a threshold effect rather than a monotonic trend.

A growing number of epidemiological studies have been published showing the association of infant feeding practices with later intake of fruit and vegetables ${ }^{(13-17)}$. To our knowledge, however, this association has not previously been described in non-Western populations. Our 
Table 2 Association between breast-feeding duration and low consumption frequency of fruit and vegetables $(<1$ time/d) at 18-24 months of age among children who participated in the OMCHS $(n 763)$

\begin{tabular}{|c|c|c|c|c|c|c|c|c|}
\hline & \multicolumn{4}{|c|}{ Fruit consumption $<1$ time $/ \mathrm{d}$} & \multicolumn{4}{|c|}{ Vegetable consumption $<1$ time/d } \\
\hline & \multicolumn{2}{|c|}{ Univariate } & \multicolumn{2}{|c|}{ Multivariate* } & \multicolumn{2}{|c|}{ Univariate } & \multicolumn{2}{|c|}{ Multivariate* } \\
\hline & OR & $95 \% \mathrm{Cl}$ & OR & $95 \% \mathrm{Cl}$ & OR & $95 \% \mathrm{Cl}$ & OR & $95 \% \mathrm{Cl}$ \\
\hline \multicolumn{9}{|l|}{ Infant feeding practices } \\
\hline \multicolumn{9}{|l|}{ Breast-feeding duration (months) } \\
\hline$<6$ & 1.00 & Reference & 1.00 & Reference & 1.00 & Reference & 1.00 & Reference \\
\hline $6-11$ & 1.00 & $0.66,1.50$ & $1 \cdot 11$ & $0.71,1.72$ & 0.48 & $0.26,0.90$ & 0.52 & $0.27,0.99$ \\
\hline $12-17$ & 0.95 & $0.64,1.41$ & 1.11 & $0.72,1.69$ & 0.56 & $0.32,0.99$ & 0.62 & $0.34,1.15$ \\
\hline$\geq 18$ & 1.00 & $0.67,1.50$ & $1 \cdot 17$ & $0.75,1.81$ & 0.55 & $0.31,1.00$ & 0.64 & $0.33,1.21$ \\
\hline \multicolumn{9}{|l|}{ Age at introduction to solid foods (months) } \\
\hline$<5$ & 1.00 & Reference & 1.00 & Reference & 1.00 & Reference & 1.00 & Reference \\
\hline $5-5.9$ & 0.71 & $0.46,1.09$ & 0.75 & $0.48,1.18$ & 0.62 & $0.33,1.14$ & 0.64 & $0.33,1.22$ \\
\hline$\geq 6$ & 0.96 & $0.62,1.48$ & 1.06 & $0.67,1.69$ & 0.77 & $0.42,1.41$ & 0.78 & $0.41,1.49$ \\
\hline \multicolumn{9}{|l|}{ Potential other factors } \\
\hline \multicolumn{9}{|l|}{ Child factors } \\
\hline Sex $($ boy $=1$, girl =0) & $1 \cdot 17$ & $0.88,1.55$ & $1 \cdot 21$ & $0.90,1.65$ & 0.80 & $0.52,1.23$ & 0.82 & $0.52,1.30$ \\
\hline Age (months) & 1.20 & $1.05,1.37$ & 1.20 & $1.05,1.39$ & 1.08 & $0.89,1.32$ & 1.05 & $0.84,1.30$ \\
\hline Birth weight ( 1 unit $=1 \mathrm{~kg}$ increase) & $1 \cdot 14$ & $0.81,1.60$ & 1.09 & $0.75,1.59$ & 0.84 & $0.50,1.41$ & 0.89 & $0.54,1.47$ \\
\hline \multicolumn{9}{|l|}{ Maternal factors } \\
\hline Maternal age at baseline (age) & 0.93 & $0.90,0.97$ & 0.95 & $0.91,0.99$ & 0.99 & $0.93,1.04$ & 1.01 & $0.95,1.07$ \\
\hline BMl at enrolment $\left(\mathrm{kg} / \mathrm{m}^{2}\right)$ & 1.02 & $0.97,1.07$ & 1.02 & $0.97,1.08$ & 1.06 & $0.99,1.14$ & 1.08 & $0.99,1.16$ \\
\hline \multicolumn{9}{|l|}{ Education (years) } \\
\hline$<13$ & 1.00 & Reference & 1.00 & Reference & 1.00 & Reference & 1.00 & Reference \\
\hline $13-14$ & 0.63 & $0.44,0.89$ & 0.64 & $0.44,0.93$ & 0.48 & $0.30,0.78$ & 0.51 & $0.31,0.85$ \\
\hline$\geq 15$ & 0.55 & $0.37,0.80$ & 0.65 & $0.43,0.98$ & 0.34 & $0.18,0.61$ & 0.41 & $0.21,0.78$ \\
\hline \multicolumn{9}{|l|}{ Family income (Japanese yen/year) } \\
\hline$<4000000$ & 1.00 & Reference & 1.00 & Reference & 1.00 & Reference & 1.00 & Reference \\
\hline $4000000-5999999$ & 0.77 & $0.54,1.10$ & 0.91 & $0.62,1.33$ & 0.68 & $0.41,1.16$ & 0.82 & $0.47,1.45$ \\
\hline$\geq 6000000$ & 0.46 & $0.31,0.67$ & 0.59 & $0.38,0.90$ & 0.83 & $0.49,1.43$ & 1.32 & $0.71,2.46$ \\
\hline \multicolumn{9}{|l|}{ Smoking during pregnancy } \\
\hline Never smoking & 1.00 & Reference & 1.00 & Reference & 1.00 & Reference & 1.00 & Reference \\
\hline Stopped smoking & 3.03 & $1.55,5.91$ & 2.49 & $1.24,5.02$ & 1.70 & $0.79,3.63$ & 1.35 & $0.60,3.03$ \\
\hline Smoked throughout pregnancy & 0.98 & $0.55,1.76$ & 0.68 & $0.37,1.28$ & 1.29 & $0.56,2.97$ & 0.80 & $0.32,2.00$ \\
\hline Fruit consumption ( 1 unit $=50 \mathrm{~g} / \mathrm{d}$ increase) & 0.83 & $0.76,0.91$ & 0.85 & $0.78,0.93$ & & - & & - \\
\hline Vegetable consumption ( 1 unit $=50 \mathrm{~g} / \mathrm{d}$ increase) & & - & & - & 0.65 & $0.55,0.78$ & 0.66 & $0.55,0.79$ \\
\hline
\end{tabular}

OMCHS, Osaka Maternal and Child Health Study.

${ }^{*}$ Adjusted for all other variables shown in the table.

finding that breast-feeding for $\geq 6$ months may reduce the risk of low intake frequency of vegetables in early childhood is consistent with the findings of previous Western studies $^{(13-17)}$. A recent analysis of four European cohorts, from the UK, France, Greece and Portugal, found that longer breast-feeding duration, regardless of exclusiveness, was consistently related to higher fruit and vegetable intake when the children were between 2 and 4 years of age ${ }^{(16)}$. Positive associations between longer exclusive breastfeeding duration and later intake of vegetables have also been observed in studies from Quebec ${ }^{(14)}$, Amsterdam ${ }^{(15)}$ and the USA ${ }^{(17)}$. In particular, the result of the Quebec study suggests a threshold effect rather than a linear trend effect, with a cut-off at 3 months of exclusive breast-feeding in relation to later vegetable intake ${ }^{(14)}$. This is similar to the apparent threshold effect at 6 months of total breast-feeding that we observed in the present study. It remains unclear whether the effect of breast-feeding on later intake of vegetables is truly a threshold effect rather than a linear trend effect because the type of breast-feeding (i.e. whether exclusive or combined with solid food) and breast-feeding duration were not categorized consistently across the available studies ${ }^{(14-16)}$. Nevertheless, it is clear that a longer duration of breast-feeding is consistently linked to higher vegetable intake later in childhood regardless of country.

In contrast, breast-feeding duration was not related to later fruit intake in the present study; this finding was inconsistent with those of previous papers ${ }^{(15,17)}$. The duration of exclusive breast-feeding and that of breastfeeding in combination with solid foods both showed linear associations with fruit intake in the previous Western studies, although these associations were not always significant ${ }^{(15)}$. The reason for this inconsistency is not clear, but may be related to differences in the food cultures of Western countries and Japan with regard to fruit in the diet. In Japan, fruit is typically considered to belong to a category of desserts with high water content, traditionally called mizu-gashi. Accordingly, fruit might be offered to young children less frequently than other foods including vegetables and rice. As observed in the present study, more than half of the children $(52.0 \%)$ did not consume fruit every day. This tendency was also observed 
in the National Nutrition Survey on Preschool Children in Japan, 2005 ${ }^{(12)}$. Further studies will be necessary to determine whether the results seen in this group would also be observed in a more representative sample of the Japanese population.

In the present study, no association was observed between age at introduction to solid foods and later intake of fruit and vegetables, although children who began eating solid foods at 5 months of age consumed significantly more vegetables than did those who began eating solid foods before 5 months of age $(P<0 \cdot 05)$. In the Avon Longitudinal Study of Pregnancy and Children (ALSPAC), later introduction to fruit appeared to be related to lower fruit intake at 2 years, but not at 3, 4, 7 or 9 years, whereas later introduction to vegetables was related to lower vegetable intake throughout childhood ${ }^{(16)}$. In a study in Amsterdam, introducing solid foods before the age of 4 months was associated with higher fruit intake compared with introducing them at 6 months, but no relationship was observed between age at introduction of solid foods and vegetable intake ${ }^{(15)}$. Another study from Quebec showed no relationship between age of introduction to vegetables and later vegetable intake ${ }^{(14)}$. Overall, the evidence regarding the relationship between age at introduction to solid foods, regardless of the types of foods initially introduced, and later intake of fruit and vegetables is currently inconclusive.

In keeping with the growing recognition of the potential effects of mothers' diets and sociodemographic factors on children's diets ${ }^{(13-15,31)}$, we found that maternal education level and fruit and vegetable intake during pregnancy were independently associated with children's intake of fruit and vegetables. Although it is not clear exactly how higher maternal education level influences children's diets, it is presumed that more educated mothers are more interested, and able, to acquire information on children's nutrition. Mothers' food choices for their children are likely determined based on the maternal knowledge and ability to understand and apply dietary recommendations, which may be connected to their education level. It is also known that maternal dietary habits during pregnancy influence children's dietary habits $^{(32)}$. Mothers who choose healthy behaviours for themselves are more likely to choose them for their children. Additionally, maternal diet influences the child's diet through chemosensory means ${ }^{(33,34)}$. Experimental research has revealed that a child's first experiences of flavour occur in utero, long before he/she is introduced to solid foods in infancy ${ }^{(33,34)}$. Flavour volatiles and taste compounds from the maternal diet during pregnancy are transmitted to the amniotic fluid and to human milk, flavouring both ${ }^{(34)}$. Thus, breast-fed infants whose mothers consumed a diet rich in fruit and/or vegetables during pregnancy may exhibit greater acceptance of the flavours of vegetables, although the present study shows no evidence that this occurs for fruit as well.
The strengths of the current study include its prospective design, the studying of a homogeneous population of Japanese mother-child pairs with similar backgrounds and our consideration of a wide range of confounding influences on the relationship between infant feeding practices and later intake of fruit and vegetables. Several limitations of the study also have to be considered, however. First, the study participants were not a representative sample of Japanese mother-child pairs in the general population. Compared with mothers who did not supply their children's dietary data, the mothers included in the analysis tended to be older, better educated, less likely to smoke during pregnancy and to have higher annual household income. Thus, the present findings might not be generalized. However, we adjusted for each of these factors in our statistical models, and unless the associations between infant feeding practices and later intake of fruit and vegetables were different in the remainder of the cohort, it is unlikely that selection bias could have been solely responsible for our findings. Second, our sample size was relatively small compared with those of previous studies ${ }^{(14-17)}$. Therefore, the statistical power may have been insufficient to allow the detection and stability of results concerning the association between infant feeding practices and later intake of fruit and vegetables. Third, the dietary data of children were obtained from a non-validated questionnaire that was developed for the OMCHS survey. The accuracy of this method for assessing consumption frequency remains a serious concern and incompleteness of the assessment cannot be ruled out. Furthermore, misreporting of self-reported food intake is a source of measurement error. This error is expected to have attenuated associations. In addition, we used a validated dietary assessment questionnaire for the mothers ${ }^{(21-27)}$, but the validity of the DHQ regarding fruit and vegetables has not been examined among pregnant women and the results should accordingly be interpreted with caution. Fourth, maternal body weight just before pregnancy was not available. We therefore used self-reported body weight collected at the baseline survey between 5 and 39 weeks of gestation. Unfortunately, this variable did not accurately reflect the usual maternal weight status, especially if it was based on maternal weight during the later stages of pregnancy. Finally, a longer duration of breast-feeding may act as a marker for more healthy behaviours of the mothers and/or highly health-conscious family influences, which could potentially confound associations with fruit and vegetable intake of children. While we controlled for a large number of potential confounding factors and other influences on fruit and vegetable intake of children, such as maternal socio-economic status and maternal dietary intake, we cannot rule out unmeasured or residual confounding in an observational study. Moreover, the validity of potential confounders from self-administered questionnaires developed for the present study is questionable because of the lack of a validation study. Therefore, the results should be interpreted with caution. 
In conclusion, the present study found that breastfeeding duration of at least 6-11 months may prevent a low intake frequency of vegetables among Japanese toddlers. As dietary habits established in early life may track from childhood to adulthood, additional longitudinal studies are needed to confirm these findings and to establish the longterm effects of early feeding practices on later dietary intake in non-Western, especially Asian, populations.

\section{Acknowledgements}

Acknowledgements: The authors thank the Neyagawa City Government, Hirakata City Government, Katano City Government, Shijonawate City Government, Kaizuka City Government, Takaishi City Government, Hannan City Government, Neyagawa City Medical Association, Hirakata City Medical Association and Kadoma City Medical Association for their valuable support. Financial support: This work was supported in part by a Grant-in-Aid (grant numbers 13770206 and 16790351) for Scientific Research from the Ministry of Education, Culture, Sports, Science and Technology and Research on Allergic Disease and Immunology from the Ministry of Health, Labour, and Welfare. The funders had no role in the design, analysis or writing of this article. Conflict of interest: Y.M. and K.T. have received funding from Meiji Co., Ltd. The other authors have indicated they have no financial relationships relevant to this article to disclose. Authorship: H.O. conducted the statistical analyses and wrote the manuscript. Y.M. contributed to the planning of the OMCHS and data collection and assisted in manuscript preparation. S.S. assisted in manuscript preparation. K.T. contributed to data collection. Y.H. supervised the design and execution of the OMCHS. All authors contributed to and have approved the final manuscript. Ethics of buman subject participation: This study was conducted according to the guidelines of the Declaration of Helsinki and approved by the ethics committee of the Osaka City University School of Medicine. Written informed consent was obtained from all participating women.

\section{Supplementary material}

To view supplementary material for this article, please visit http://dx.doi.org/10.1017/S1368980015001779

\section{References}

1. Robinson S \& Fall C (2012) Infant nutrition and later health: a review of current evidence. Nutrients 4, 859-874.

2. Przyrembel H (2012) Timing of introduction of complementary food: short and long-term health consequences. Ann Nutr Metab 60, 8-20.

3. Mennella JA \& Trabulsi JC (2012) Complementary foods and flavor experiences: setting the foundation. Ann Nutr Metab 60, $40-50$.

4. Schwartz C, Scholtens PA, Lalanne A et al. (2011) Development of healthy eating habits early in life. Review of recent evidence and selected guidelines. Appetite $\mathbf{5 7}$, 796-807.

5. Devine CM, Connors M \& Bisogni JS (1998) Life-course influences on fruit and vegetable trajectories. Qualitative analysis of food choices. J Nutr Educ 30, 361-370.

6. Maynard M, Gunnell D, Emmett P et al. (2003) Fruit, vegetables, and antioxidants in childhood and risk of adult cancer: the Boyd Orr cohort. J Epidemiol Community Health 57, 218-225.

7. Ness AR, Maynard M, Frankel S et al. (2005) Diet in childhood and adult cardiovascular and all cause mortality: the Boyd Orr cohort. Heart 91, 894-898.

8. Mikkilä V, Räsänen L, Raitakari OT et al. (2005) Consistent dietary patterns identified from childhood to adulthood: the Cardiovascular Risk in Young Finns Study. Br J Nutr 93, 923-931.

9. Manios Y, Kourlaba G, Kondaki K et al. (2009) Diet quality of preschoolers in Greece based on the Healthy Eating Index: the GENESIS study. J Am Diet Assoc 109, 616-623.

10. Lioret S, Dubuisson C, Dufour A et al. (2010) Trends in food intake in French children from 1999 to 2007: results from the INCA (étude Individuelle Nationale des Consommations Alimentaires) dietary surveys. Br J Nutr 103, 585-601.

11. Krebs-Smith SM, Guenther PM, Subar AF et al. (2010) Americans do not meet federal dietary recommendations. J Nutr 140, 1832-1838.

12. Ministry of Health, Labour, and Welfare of Japan (2006) National Nutrition Survey on Preschool Children in Japan, 2005 (in Japanese). http://www.mhlw.go.jp/houdou/2006 /06/dl/h0629-1b.pdf (accessed March 2015).

13. Cooke LJ, Wardle J, Gibson EL et al. (2004) Demographic, familial and trait predictors of fruit and vegetable consumption by pre-school children. Public Health Nutr $\mathbf{7}$, 295-302.

14. Burnier D, Dubois L \& Girard M (2011) Exclusive breastfeeding duration and later intake of vegetables in preschool children. Eur J Clin Nutr 65, 196-202.

15. Möller LM, de Hoog ML, van Eijsden M et al. (2013) Infant nutrition in relation to eating behaviour and fruit and vegetable intake at age 5 years. BrJ Nutr 109, 564-571.

16. de Lauzon-Guillain B, Jones L, Oliveira A et al. (2013) The influence of early feeding practices on fruit and vegetable intake among preschool children in 4 European birth cohorts. Am J Clin Nutr 98, 804-812.

17. Perrine CG, Galuska DA, Thompson FE et al. (2014) Breastfeeding duration is associated with child diet at 6 years. Pediatrics 134, Suppl. 1, S50-S55.

18. Mennella JA, Lukasewycz LD, Castor SM et al. (2011) The timing and duration of a sensitive period in human flavor learning: a randomized trial. Am J Clin Nutr 93, 1019-1024.

19. Miyake Y, Miyamoto S, Ohya Y et al. (2004) Relationship between active and passive smoking and total serum IgE levels in Japanese women: baseline data from the Osaka Maternal and Child Health Study. Int Arch Allergy Immunol 135, 221-228.

20. Miyake Y, Sasaki S, Tanaka K et al. (2006) Dietary folate and vitamins $\mathrm{B}_{12}, \mathrm{~B}_{6}$, and $\mathrm{B}_{2}$ intake and the risk of postpartum depression in Japan: the Osaka Maternal and Child Health Study. J Affect Disord 96, 133-138.

21. Sasaki S, Yanagibori R \& Amano K (1998) Self-administered diet history questionnaire developed for health education: a relative validation of the test-version by comparison with 3-d diet record in women. J Epidemiol 8, 203-215.

22. Sasaki S, Yanagibori R \& Amano K (1998) Validity of a selfadministered diet history questionnaire for assessment of sodium and potassium: comparison with single 24-hour urinary excretion. Jpn Circ J 62, 431-435.

23. Sasaki S, Ushio F, Amano K et al. (2000) Serum biomarkerbased validation of a self-administered diet history questionnaire for Japanese subjects. J Nutr Sci Vitaminol (Tokyo) 46, 285-296. 
24. Kobayashi S, Murakami K, Sasaki S et al. (2011) Comparison of relative validity of food group intakes estimated by comprehensive and brief-type self-administered diet history questionnaires against $16 \mathrm{~d}$ dietary records in Japanese adults. Public Health Nutr 14, 1200-1211.

25. Kobayashi S, Honda S, Murakami K et al. (2012) Both comprehensive and brief self-administered diet history questionnaires satisfactorily rank nutrient intakes in Japanese adults. J Epidemiol 22, 151-159.

26. Shiraishi M, Haruna M, Matsuzaki M et al. (2012) Validity and reproducibility of folate and vitamin $\mathrm{B}(12)$ intakes estimated from a self-administered diet history questionnaire in Japanese pregnant women. Nutr J 11, 15.

27. Shiraishi M, Haruna M, Matsuzaki M et al. (2013) Validity of a diet history questionnaire estimating $\beta$-carotene, vitamin $\mathrm{C}$ and $\alpha$-tocopherol intakes in Japanese pregnant women. Int J Food Sci Nutr 64, 694-699.

28. Science and Technology Agency (2010) Standard Tables of Food Composition in Japan 2010 (in Japanese). Tokyo: Printing Bureau of the Ministry of Finance.

29. Ministry of Health, Labour, and Welfare of Japan (2007) Support Guide of the Nursing and Weaning (in Japanese). http://www.mhlw.go.jp/shingi/2007/03/s0314-17.html (accessed March 2015).

30. Okubo H, Miyake Y, Sasaki S et al. (2014) Dietary patterns in infancy and their associations with maternal socioeconomic and lifestyle factors among 758 Japanese mother-child pairs: the Osaka Maternal and Child Health Study. Matern Child Nutr 10, 213-225.

31. North K \& Emmett P (2000) Multivariate analysis of diet among three-year-old children and associations with sociodemographic characteristics. The Avon Longitudinal Study of Pregnancy and Childhood (ALSPAC) Study Team. Eur J Clin Nutr 54, 73-80.

32. Robinson S, Marriott L, Poole J et al. (2007) Dietary patterns in infancy: the importance of maternal and family influences on feeding practice. Br J Nutr 98, 1029-1037.

33. Mennella JA (2007) The chemical senses and the development of flavor preferences in humans. In Textbook on Human Lactation, pp. 403-414 [PE Hartmann and T Hale, editors]. Amarillo, TX: Hale Publishing.

34. Beauchamp GK \& Mennella JA (2009) Early flavor learning and its impact on later feeding behavior. J Pediatr Gastroenterol Nutr 48, Suppl. 1, S25-S30. 